\title{
ROLE PATTERNS IN IT PROJECTS TEAMS: DESIGN OF A SELECTION MODULE USING FUZZY LOGIC TECHNIQUES
}

\author{
Irena BACH-DĄBROWSKA*, Jakub WOJNAR** \\ Department of Management and Economy, Gdańsk University of Technology, Gdańsk, Poland \\ *e-mail: ibach@zie.pg.gda.pl \\ **e-mail: jakub.wojnar@gmail.com
}

\begin{abstract}
This paper introduces an approach based on role patterns and modelling by the use of fuzzy logic tool for selection process with limitation to the area of IT projects environment. The article shows a concept of role pattern structures and their further usage in process of forming a fuzzy model dedicated to candidate assessment process support.
\end{abstract}

Key words: selection process, role patterns, fuzzy logic techniques, MBIT technique.

\section{$1 \quad$ Introduction}

This paper introduces an approach based on role patterns and modelling by the use of fuzzy logic tool for selection process with limitation to the area of IT projects environment. This limitation follows the impact caused on team work and team structure by specific models and methods implemented in the IT project management. Popularity of a new, agile philosophy imposed a new perspective and ideas of a team work and introduction of new requirements, alternative to classical project management standards in this field relating to the characteristic of team members.

\section{Teams of IT projects}

An analysis of current sources of the industry literature $[3-5,9,10]$ indicates noticeable process of accomplishing change in an organisational structure of IT project teams conducted in agile methodologies, as well as in happening changes due to requirements put in front of candidates to such teams.

First of all, agile teams of IT projects lose their vertical structure of hierarchy, where a clear subjection and dependence of positions existed. An agile team, by its definition, is a team of equal partners who are supposed to do tasks and make decisions collectively. In the foregoing defined role hierarchy, there is a structure of group superiority, which is characterised by different level of coherence (strength of bonds between members of the team). Not every person is competent to work in such an environment, as not everyone is able to find their place in a group where the role range fades. There is a possibility that people with strong ego and strong leading features will not manage well in the environment of agile projects, because such people will aim to force their ideas and opinions through with leaving no space for compromises and commonly worked out solutions. Also, people with strong introversive characteristics can feel inconvenient in a group functioning that way, because one of the basic work rule of agile teams is running everyday meetings that are based on brainstorm techniques, which requires every member of a group to be engaged in conversations, to report work progress, to talk through problems encountered during tasks executing and collective working through solutions and deciding about the direction of a project development.

Another change is a size of a group. Big, stratified project team very often consisting of over 20 people is changed into 8-10 people team which is a mixture of different personalities and competencies. Depletion of a size team brings the change of requirements of knowledge range of each team member and emphasises complementarity of skills of a team as an entire unit. Team members should have a characteristic of versatility in the range of information technology knowledge, which means that narrowly specialized workers (so called type I) are less willingly employed, and workers type $\mathrm{T}$ are employed instead of them. It is expected that versatile people will programme one day, make analysis another day and finally test created piece of programme. Additionally, teams built that way are to guarantee the support between team members in tasks carried out individually and in solving possible problems. Such tendencies are noticeable even at the level of qualifying tests for any technical post in an IT team, when the candidate must prove their command of technologies and methodologies in every possible specialisation groups of IT branch, not only with regard to the post they apply for. 
Another characteristic of agile teams is to work in doubtful conditions, which means that commencement of the project is independent of amount of possessed information and its range of changes flexibly according to needs of the client who orders a project. In practice, it means frequently appearing stressful situations and the necessity of reacting fast to changes.

All of these factors affect significantly the way and effectiveness of the team project work, management of such a team and most of all put new requirements in relation to recruitment process and employees selection. Recruitment process and employees selection of IT departments is strongly formalised and in most cases divided into stages encompassing two levels of a job interview: hard competencies tests (practical knowledge and ability to apply it) and at the end negotiating employment conditions. In this whole process, there is no place for evaluation of an adjustment of a given candidate to already existing work team. It is not common to encounter the practices enabling evaluation of the level of coherence of a team as a whole. One may believe that this element is skipped at this level because of the technical difficulties - most companies do not run databases containing personal profiles of current employees, most certainly nobody conducts systematic researches on how the level of hard and soft competencies changes with regard to particular person during the employment. Simultaneously, there are no tools supporting recruitment process, excluding obvious application forms. Secondly, with regard to technical workers, at the level of recruitment, the aspect of soft competencies evaluation and worker's behavioural profile is excluded, adopting the assumption that the person on technical post should, most of all, present a high level of technical knowledge and also practical experience.

Such way of thinking is not incorrect if one takes into consideration the tasks execution effectiveness of a particular worker who has to do one specific work type. Nevertheless, in case of IT projects, work of a particular person is tightly related to cooperation with other team members (communication, ability to solve contentious situations, negotiating) and also the ability to convey information. Actually, every standard of a project management (not only IT project) indicates relation between particular roles in a project, and not only in hierarchical dependence exclusively. The range of tasks execution and also the area of cooperation with other (chosen) team roles are usually established for a given role. Obviously, the strength and cooperation range between particular team members differ between each other.
Additionally, IT projects based on light or agile methodologies need a lot of client's engagement (his representative or the whole implementation team on the client's side) which transfers into the necessity to own high communication skills by the team workers of a deliverer of information technology solutions. The research of IT project conducted in Poland in 2010 [12] shows that success of project execution in Poland depend mostly on a client's engagement into the project execution and also on ability of cooperation between the team of an information technology deliverer and a client's team.

Above specific characteristics of teams of IT projects, at the level of completing, determine the need to provide double evaluation unit: determining project team coherence, which could be called 'team matching' in colloquial language, and also matching individual team members to the group. Execution of the research at the level of choosing single workers to a team, as well as at the level of reorganising the team allows to reduce the risk of ineffective work and the risk of ongoing work in the atmosphere of conflict, and as a result allows to moderate the process of adjusting team members to each other (which is one of four typical stages of a team development).

\section{Team's coherence and role patterns}

There are five basic features for every small group [6], that is:

- every group has an aim; in order to achieve it, group members interact with each other (information flow, cooperation),

- safeguarding of a proper interaction requires arranging the structure of group's relationship hierarchy which manifests various stages of coherence (the strength of group members' bonds),

- the fifth feature of a group is its development; every group goes through a series of stages in a process of creation, functioning and its presumptive dissolution.

At the foundation of the research lies a postulate assuming that group's coherence will be described and assessed with the usage of two variables, id est interaction strength among group members (depending on established position's patterns defining the structure of group's relationship) and the level of topological matching of group members interacting with each other strongly (the meaning and suitability of a 'strong' interaction will be defined in further parts of this paper).

The strength of interaction is defined and understood as imposed (determined by team's roles) strength of relations 
among the team's members assigned to specified project roles in the team. This strength is determined by relational position's patterns designed for all team's roles based on a specified standard of conducting IT projects.

The level of topological matching will be specified based on psychological tool id est Myers-Briggs Type Indicator (MBTI) pattern allowing to establish personal type of every individual in a team as well as the team as a whole. The level of topological matching will be correlated accurately to topological role pattern and topological-relational role pattern.

The topological position's pattern indicates what personal type (out of 16 identified in MBTI methodology) is the most or the least appropriate for specified project role, taking the kind of soft or hard competencies characteristic for each of the roles as a criterion.

The topological-relational position's pattern indicates what personal type should be manifested by people cooperating with each other in a team whose relational position shows high interaction strength.

On the basis of the pre-suppositions mentioned above the following task has been taken:

\section{Problem defining}

An IT project $P$, run in accordance with specified IT standard, is given. A project team described by set of project roles $R=\left\{r_{1}, r_{2}, \ldots, r_{n}\right\}$ is given, to which a worker is matched according to the need of a particular role. A group of recruited candidates $K=\left\{k_{1}, k_{2}, \ldots, k_{n}\right\}$ meeting primary criteria (specified technical skills and experience) is given. Role patterns matrixes are defined (relational $A_{R W S}$, topological $A_{T W S}$ and topological-relational $A_{T R W S}$ ) for specified roles in the project. One searches for an answer to a question: Is there a candidate (set of candidates) matching well to the project team, taking team's coherence as a criterion? If yes, then: Which of the candidates fits in the team's structure the most, taking team's coherence as a criterion?

The subsequent points present an approach to a structure of a module selection that allows to execute the task defined above based on a fuzzy logic modelling tool and MBTI method.

\section{Role patterns structure}

In a defined candidate to project team selecting task an assumption is taken that execution of IT project is to be based on standards of running an IT projects. Each standard has defined a range of project roles for which a set of tasks and competences for an effective accomplishment is circumscribed. A team's work is found on interactions of individuals playing specific roles in the project. The strength of these interactions influences the effective execution of assignments. In research the strength of interactions was divided into three ranges taking into consideration the strength of cooperation between various roles and its impact on the effectiveness of tasks realisation. The following groups were distinguished: lack or poor interaction, moderate interaction and strong interaction. Given ranges of strength of interactions ought to be interpreted as follows. Lack or poor interaction is characteristic for a pair of roles in which cooperation in project team does not occur or occurs sporadically and has no effect on quality/effectiveness of tasks realisation. Moderate interaction is characteristic for pair of roles in which cooperation occurs but it has got no significant effect on quality/effectiveness of project tasks realisation. Strong interaction is characteristic for pair of roles in which cooperation reaches high level and has got significant effect on quality/effectiveness of project tasks realisation. In order to be able to group these interaction strength values, it is necessary to build a relational role patterns.

\subsection{Relational role patterns}

A relational role pattern describes the strength of interaction among project team roles. The strength of interaction among roles is determined, inter alia, by the specificity of a team management method, methodology of leading the project and thus by specific project team's structure. Simultaneously, the strength of interaction may be influenced by additional factors resulting from, e.g. informal division of power in a team developed in process of collaboration (workers choose their team leaders on their own and use older ones experience regardless of their position in a team). It is vital to emphasise that relational roles patterns should be established accordingly to given organisation's specificity, because single pattern suitable for all IT environment projects does not exist.

A relational roles pattern is formed in process of assigning individual pairs of roles $p r_{i}$ to strength of interaction through series of comparisons (in pairs) of separate roles. This leads to a creation of relational roles pattern's square matrix $A_{W R S}$. The relational pattern is presented as matrix $A_{W R S}$ sized $n \times n$, where $n$ represents number of roles in given team model. 


$$
A_{W R S}=\left[\begin{array}{ccc}
0 & \ldots & a_{1 n} \\
\ldots & 0 & \ldots \\
a_{1 n} & \ldots & 0
\end{array}\right]
$$

Diagonal values $a_{i, j}=0$, for $i, j=1 \ldots n$, where $i=j$.

Values $a_{i j}$ of matrix $A_{W R S}$ placed above diagonal are ratings from range of 0 to 1 (accurate to within decimal number) attributing 0 to the poorest interaction (people with comparable roles do not have to collaborate) and 1 to the strongest interactions (people with comparable roles strongly collaborate with each other).

$$
0 \leq a_{i, j} \leq 1
$$

for where $i, j=1 \ldots n$.

Values aji placed below diagonal of the matrix equal values aij placed above the diagonal.

$$
a_{j, i}=a_{i, j}
$$

for where $i, j=1 \ldots n$.

It is assumed that interaction strength evaluation is performed by a group of domain experts $E=\left\{e_{1}, \ldots, e_{m}\right\}$ (management staff of IT teams, long-time IT teams workers) who establish relational patterns for specific methodology. It is crucial to have the rating evaluated by a group of experts of not less than five members. In that case a standardisation, relational role pattern is necessary throughout the process of averaging experts' evaluation results.

$$
\frac{1}{m} \sum_{k=1}^{m} a_{i j}^{k}
$$

for $i, j=1 \ldots n, i \neq j, k=1 \ldots m$.

\subsection{Topological role patterns}

Topological role pattern defines advisable (suggested) personality type $w t_{i}$, for $i=1 \ldots 16$, for a given team role, accepting a criterion of demanded hard and soft competences corresponding to the role. In the first place this element must be explained in context of accepted personality types (personality models) ratings of candidates and team members.

\subsubsection{Personal models according to Myers-Briggs Type Indicator (MBTI)}

There are many personality models such as Five Factor Model, Big Five, Keirsey Temperament Sorter, 16PF or
DiSC Personality Profile Assessment, but one of the most world-wide known and used is MBTI [7]. It is based on Carl Jung's theory of psychological type and it assumes that every person has natural preference in perceiving the world and making judgments. Figure 1 shows the basic schema of how people perceive the world and how they make decisions according to Jung.

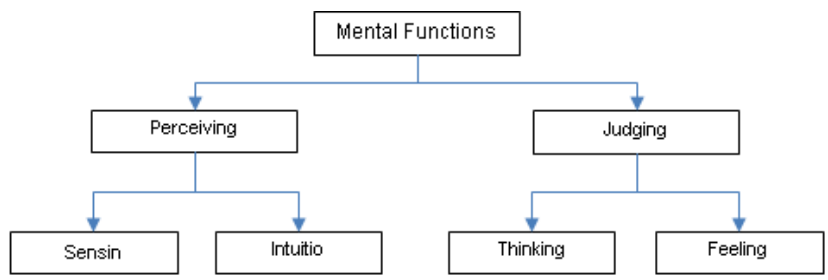

Figure 1. Partition of Mental Functions

People may perceive outer world using their five senses or by intuition when they are unconsciously incorporating and associating ideas coming from outside. With the same approach they can make decisions using logical and impersonated process or subjective approach taking into account personal outcome of the decision.

According to Cakrt, people who prefer to gather information by using all the senses need facts and practical information which they remember much easier than relationship between them or opportunities arising from them. They use their or others' experience as a base for current and future actions. Sensing people work systematically and continuously and they try not to interfere with sudden acts of inspiration or creativity. They are focused on numbers rather than theories and prefer tasks which have tangible end results rather than conceptual divagations.

On the opposite scale, people who are prefer using their intuition in the process of perceiving the world. They are mostly interested in ideas, concepts and possibilities. At the each stage of the work, they have the big picture in mind often forgetting the details. They usually try different ways of approaching the same problem just to check which one is better. They seek relations between different facts and try to understand the meaning behind the obvious statements [2].

When it comes to processing information and making decision, Jung divided people into two categories: ones that follow impersonated logical process using thinking approach and ones that rely on their feelings taking into consideration other people impacted by the outcome of particular decision.

Table 1 summarises the main differences between thinkers and feelers. 
Table 1. Main differences between Thinker and Feeler [2]

\begin{tabular}{|c|c|c|}
\hline & Thinker & Feeler \\
\hline Is focused on & Logical parameters of the problem, rules, truth & Human values, harmony, emotions \\
\hline Relies on & Past, presence, future & Past \\
\hline At work is & Impersonated, well organised, result oriented & $\begin{array}{c}\text { Friendly, interested in others, taking issues } \\
\text { personally }\end{array}$ \\
\hline Strengths are & Toughness, rationalism, logical thinking & Loyalty, empathy, personal involvement \\
\hline Possible weaknesses are & Too analytical, emotionless, rigid & $\begin{array}{c}\text { Sentimental, avoiding conflicts and } \\
\text { confrontations }\end{array}$ \\
\hline
\end{tabular}

Another dimension where Jung observed dichotomous preferences in people's behaviour was the vital energy orientation. He distinguished two groups based on a fact that if a person takes the energy from the inner world of concepts and ideas or from the outer world of people and actions.

A person with extroverted preference is characterised by general openness to the world and other people. Such person is open and friendly and has a lot of people around. Takes on new tasks and adventures with pleasure and visibly expresses all emotions. Prefers open communication and usually by talking. Extroverted person is very sociable and learns best by doing and discussing. On the other hand an introverted person is very private and contained. Prefers communication in writing and learns best by reflection and theoretical approach. Introverted person takes initiative seldom unless the issue is very important to him or her (see Figure 2).

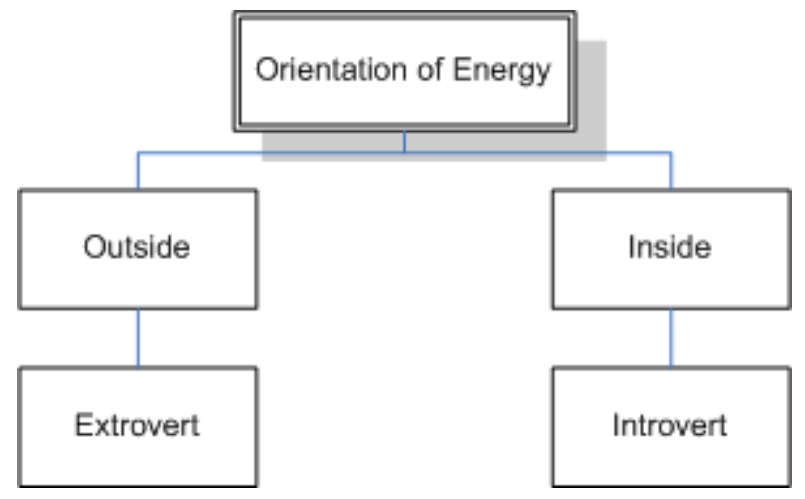

Figure 2. Energy orientation dichotomy

The last functional dichotomy that completes the four dimensions of personality type is the judgment-perception preference. Within this dimension people differ on attitude toward the way they deal with the outer world. People who prefer judging attitude usually are systematic, methodical and organised in their lives. They make short- and longterm plans in order to avoid decision not be made and issues not decided upon. On the opposite scale are people with observing preference. They are usually spontaneous, casual and flexible. They quickly and easily adapt to changes and open and energised when many things are happening at once.

Based on the above dimensions, personality type is defined by four pairs of dichotomous attributes: Extroversion/Introversion, Sensing/iNtuition, Thinking/Feeling and Perceiving/Judging. Combination of one attribute from each pair creates sixteen psychological types $w t_{i}$ that a person can be described by and they are listed in Table 2 .

Table 2. MBTI Personality Types

\begin{tabular}{|l|l|l|l|}
\hline ISTJ & ISFJ & INFJ & INTJ \\
\hline ISTP & ISFP & INFP & INTP \\
\hline ESTP & ESFP & ENFP & ENTP \\
\hline ESTJ & ESFJ & ENFJ & ENTJ \\
\hline
\end{tabular}

Each type shows such preference. For example a person characterised by type ISTJ is rather introvert that collects data by sensing makes decision by logical analysis and prefers systematic and planned way of acting.

In order to analyse and create the desired team structure, each of the MBTI types is decomposed in single attributes and those attributes are valued from the perspective of cooperation capabilities [11].

This task is assigned to psychology experts whose expertise and experience allow to build suitable topological role patterns for specific work environment and team roles characterised by indicated group of competence expectations.

The structure of topological role pattern consists in assigning individual project roles $r_{i}$ to preferred topological pattern $w t_{i}$. A group of work psychology experts $E p=$ $\left\{e p_{1}, \ldots, e p_{1}\right\}$ assigns each topological pattern $w t_{i}$ to rating from 0 to 1 (in range of decimal values) judging its 
adequacy for given project role. This creates a matrix of topological role pattern $A_{T W S}$ sized $n \times m$, where $n$ stands for number of roles in specific role model and $m$ number of personality types judged in context of position roles.

$$
A_{T W S}=\left[\begin{array}{ccc}
a_{11} & \ldots & a_{1 n} \\
\ldots & \ldots & \ldots \\
a_{m 1} & \ldots & a_{m n}
\end{array}\right]
$$

where $0 \leq a_{i, j} \leq 1$, for $i=1 \ldots n, j=1 \ldots m$. Diagonal values $a_{i, j} \neq 0$, for $i=j$.

Values $a_{j i}$ placed below diagonal of the matrix differs from values $a_{i j}$ placed above the diagonal.

$$
a_{j, i} \neq a_{i, j}
$$

for where $i=1 \ldots n, j=1 \ldots m$.

It is crucial to have the rating evaluated by a group of experts of not less than five members. In that case a standardisation of relational role pattern is necessary through a process of averaging experts' evaluation results.

$$
\frac{1}{l} \sum_{k=1}^{l} a_{i j}^{k}
$$

for $i=1 \ldots n, j=1 \ldots m, k=1 \ldots l$.

\subsection{Topological-relational role patterns}

From previous researches, it has been presumed that topological matching people to team roles will be important for those combinations of roles which remain in a strong interaction, which is why topological-relational role patterns are built only for those role pairs that are allocated to this level of interaction strength group.

Topological-relational role patterns define indicated personality type preferences for pair combined comparisons from the group of a high level of interaction. In other words it is elucidated what personality types ought to (may) effectively cooperate with each other and which ones should not be teamed. This task is again assigned to psychology expert's opinion.

The form of topological-relational role pattern consists in assigning (defining) respective pairs of roles from a strong interaction group to topological matching rating taking into account the effectiveness (amicability) of collaboration. A group of work psychology experts $E p=$ $\left\{e p_{1}, \ldots, e p_{1}\right\}$ assigns each relational pair from strong interaction group to a rating from 0 to 1 (in a range of decimal values) stipulating capability of cooperation for all personality types put in pairs. It results in creation of topological-relational role pattern's matrix $A_{T R W S}$ sized $n \times n$, where $n$ stands for number of personality types according to MBTI.

$$
A_{T R W S}=\left[\begin{array}{ccc}
a_{11} & \ldots & a_{1 n} \\
\ldots & \ldots & \ldots \\
a_{n 1} & \ldots & a_{n n}
\end{array}\right]
$$

where $0 \leq a_{i j} \leq 1$, for $i, j=1 \ldots n$.

It is crucial to have the rating evaluated by a group of experts of not less than five members. In that case a standardisation of relational role pattern is necessary through a process of averaging experts' evaluation results according to formula (7).

Created role patterns are foundation for comparison of candidate's score obtained in process of selection for proper project role to expected (perfect) results defined by domain experts for a given enterprise.

Possibility of applying those patterns requires building an assessment instrument for evaluating and ranking verified candidates according to arranged rules. It is ought to be emphasised that all patterns (matching rules) are built based on an expert's knowledge. All the scores also involve quality evaluation of so-called soft aspects of person's competencies, which requires applying approximate and intuitive ratings burdened with each domain expert's subjective point of view based on linguistic evaluation. Those features fit in characteristics of fuzzy logic instrument in which conducted research is one of the chosen methods for description of knowledge necessary in a selection process.

\section{$5 \quad$ Fuzzy modelling based on system expert's knowledge}

Modelling based on system expert's knowledge (domain expert) is built on experience and expertise of a person who knows the system well. In considered case of role patterns structure domain experts from IT group and occupational psychology are employed. Experts' knowledge has a dual character - open (conscious expertise) and unconscious (intuitional expertise, so called 'hunch'). Open knowledge may be expressed in words and passed to another person, whereas unconscious knowledge is difficult to be enunciated (described). Combination of the two above mentioned kinds of knowledge about reality system in expert's mind is defined as mental or mind model [1]. In process of knowledge extraction from system expert only open expertise is acquired, which is manifested as 


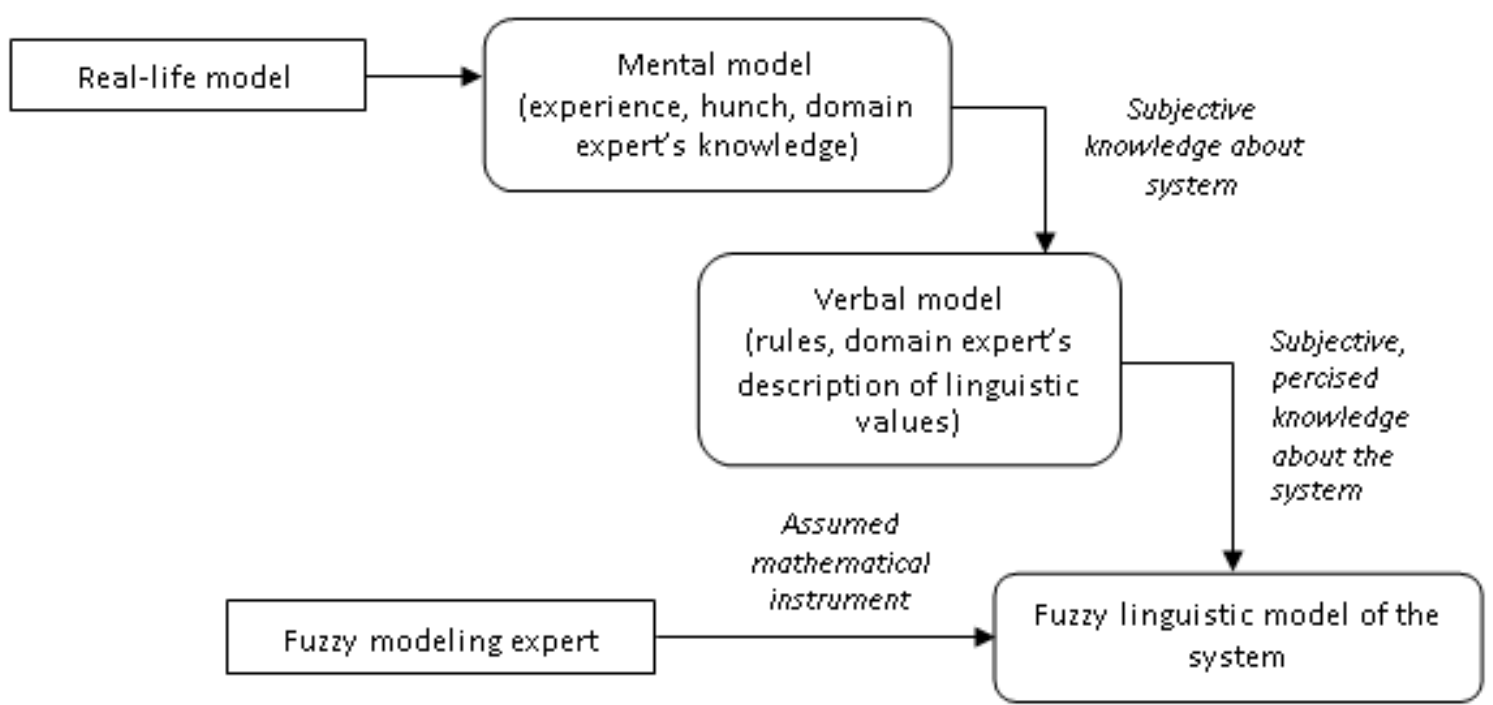

Figure 3. The creation process of real-life system fuzzy linguistic model [8]

word rules indicating enter/exit relationship type:

$$
\operatorname{IF}\left(x_{1} \text { if } A_{i}\right) \operatorname{AND}\left(x_{2} \text { if } B_{j}\right) \text { THEN }\left(y \text { is } C_{k}\right)
$$

where: $x_{1}, x_{2}$-system's input; $y$-system's output; $A_{i}, B_{j}$, $C_{k}$ - fuzzy sets used for linguistic evaluation of a system output by an expert.

Rules exemplification: IF (the team is experienced) AND (the project is well defined) THEN (meeting deadline is possible). System expert also assesses the way of valuation of used linguistic ratings through particularising labels and value ranges, for example 'experienced team' stands for a team that has worked together on projects for at least 5 years.

A set of verbal rules defining system's input/output relationship and a set of verbal information about linguistic value used by an expert is called system's verbal model. Verbal model is usually flatter than mental model, because the first one does not include intuitional knowledge which is not possible for an expert to pass. The system expert is also not able to get across the knowledge about illation mechanisms taking place in his mind, about a kind (form) of membership function describing linguistic values, about kind of logical operators used in process of metal data processing, etc.

All that information necessary for fuzzy linguistic model creation of a given system must be supplemented supposedly (intuitively) by a modeller, who can be termed fuzzy modelling expert [8].

Information flow that takes place in the process of the system fuzzy, linguistic model creation is presented in Figure 3.

\section{$6 \quad$ Fuzzy model structure}

Fuzzy logic instrument is based on a group of primary concepts allowing the model knowledge about described systems. Hereunder there are main definitions in accordance with research literature [8].

Linguistic variable - this input/output figure or state variable, which is evaluated by linguistic rating, called linguistic values. For example: ship's speed, tension, temperature.

Linguistic value - is a verbal value of linguistic figure. For example: very big, average, old, young.

Variable linguistic space - is a set of all linguistic values used for assessment of a given linguistic variable. It is lettered in capital Latin characters, e.g.: $X_{\mathrm{L}}=\{$ negative, positive $\}=\left\{x_{\mathrm{L} 1}, x_{\mathrm{L} 2}\right\}$.

The linguistic space is a finite dimensional set.

Variable numeric space (consideration space) - is all numeric values set that can be taken by the set in considered system or such values which are vital for problem solving (model system). Variable numeric space is lettered in capital Latin characters, for example:

- $X=\{x\}-$ infinitely dimensional space, e.g. $x \in \mathbb{R}$

- $X=\left\{x_{1}, x_{2}, \ldots, x_{n}\right\}$ - finitely dimensional space, discreet, e.g. $\left\{x_{1}=0.75, x_{2}=1\right\}$

Fuzzy set. A fuzzy set $A$, in some numeric space of consideration $X$ is called a pair set:

$$
A=\left\{\left(\mu_{A}^{*}(x), x\right)\right\}
$$

where: $\mu_{A}$ is membership function of fuzzy set $A$ which associates each element $x \in X$ to a membership grade 
$\mu_{A}^{*}(x)$ to fuzzy set $A$, when: $\mu_{A}(x) \in[0,1]$. Membership function models numeric space $X$ of a given variable to a range $[0,1]$ :

$$
\mu_{A}: X \rightarrow[0,1]
$$

Fuzzy modelling expert's role is competent choice of method of obtained knowledge representation to modelled phenomenon (range of knowledge). With limited spectrum of information about modelled system a polygonal function is used. In order to model this function a minimal amount of information is required [data concerning modal functions points (minimum, average, maximum)]. Simultaneously carried out researches, concerning mainly evaluative decisions, show that a person uses (often subconsciously) so called intuitive functions which are continuous in numeric consideration space which means that any small change of observed variable $x$ does not cause abrupt change of speed of this variable rating (quality rating). In case of polygonal functions abrupt changes are characteristic feature which indicates that those functions are good approximation of human way of judging. That is the reason why in the process of building a model of human's psychological assessment function, intuitional function will be applied, including Gauss's symmetric function with critical point for modelling internal fuzzy sets and sigmoidal functions of fixture to external sets representation.

\subsection{Fuzzy model for assessment of interaction strength among roles in team}

The model for assessment of interaction strength among roles in team is built in SISO (single input, single output) system, where with an input of a system variable $x_{R W S}$ is given - cooperation level of project role pair $p r_{i}$, for which:

- linguistic space $X_{\mathrm{L}}$ of input variable $x_{R W S}:\left\{A_{1}-\right.$ low, $A_{2}-$ average, $A_{3}-$ high ,

- numeric space $X_{\mathrm{N}}$ of input variable $x_{R W S}:\left\{x_{R W S} \in \mathbb{R}, 0 \leq\right.$ $\left.x_{R W S} \leq 1\right\}$,

and system output result in - interaction strength of project role pair, for which:

- linguistic space $Y_{\mathrm{L}}$ of output variable $y_{R W S}:\left\{B_{1}-\right.$ lack or low, $B_{2}$ - average, $B_{3}-$ high ,

- numeric space $Y_{\mathrm{N}}$ of output variable $y_{R W S}:\left\{y_{R W S} \in \mathbb{R}, 0 \leq\right.$ $\left.y_{R W S} \leq 1\right\}$,

It is assumed that sets $A_{i}$ and $B_{i}$ are described by following functions:
- for system input

$$
\begin{aligned}
\mu_{A 1}\left(x_{R W S}\right)= & \frac{1}{1+e^{-a\left(x_{R W S}-b\right)},} \\
\mu_{A 2}\left(x_{R W S}\right)= & e^{-\left(\frac{x_{R W S}-b}{a}\right)^{2}}, \\
& \mu\left(x_{R W S_{k 1}}\right)=0.5, \\
& \mu\left(x_{R W S_{k 2}}\right)=0.5 \\
\mu_{A 3}\left(x_{R W S}\right)= & \frac{e^{-a\left(x_{R W S}-b\right)}}{1+e^{-a\left(x_{R W S}-b\right)}}
\end{aligned}
$$

- for system output

$$
\begin{gathered}
A_{R W S}=\left[\begin{array}{ccc}
0 & \ldots & a_{1 n} \\
\ldots & 0 & \ldots \\
a_{n 1} & \ldots & 0
\end{array}\right] \Longrightarrow \\
\mu_{C 1}\left(y_{R W S}\right)=\frac{1}{1+e^{-a\left(y_{R W S}-b\right)}}, \\
\mu_{C 2}\left(y_{R W S}\right)=e^{-\left(\frac{y_{R W S}-b}{a}\right)^{2}} \\
\mu\left(y_{R W S}\right)=0.5 \\
\mu\left(y_{R W S}\right)=0.5 \\
\mu_{C 3}\left(y_{R W S}\right)=\frac{e^{-a\left(y_{R W S}-b\right)}}{1+e^{-a\left(y_{R W S}-b\right)}}
\end{gathered}
$$

In a process of modelling the system output sets $y_{R W S}$ a standardised pattern for positioning relation $A_{R W S}$ is used. Values received from experts assessments are assorted to three linguistic sets $B_{1}, B_{2}, B_{3}$, accepting rating range from 0 to 1 (e.g. $y_{R W S_{B_{1}}}[0 \div 0.5], y_{R W S_{B_{2}}}[0.3 \div 0.8]$ and $\left.y_{R W S_{B}}[0.5 \div 1]\right)$.

Additionally the internal function's description (Gauss' function) both system input and output is broadened by notion of critical point $k$ of membership function that allows to indicate such point of this function in which membership level equals 0.5 . Introducing critical point $k$ is a measure that enables defining basic set characteristic points which membership level equals 0.5. Contemporaneously an assumption is accepted that adjacent (outer) membership functions will intersect with Gauss' function at points $x_{k_{1}}, x_{k_{2}}$ and $y_{k_{1}}, y_{k_{2}}$, and hence critical points will be set for those coordinates which do not clearly belong to either outer or inner fuzzy set.

A knowledge base is represented as If-Then type of rule including all combinations of input variable values and respective set of conclusions.

$$
\text { IF } x_{R W S} \text { IS } A_{i} \text { THEN } y_{R W S} \text { IS } B_{i}
$$

Example 1:

Project $P$, in which the following set of project roles appears $R=\left\{r_{1}, r_{2}, r_{3}, r_{4}, r_{5}, r_{6}\right\}$, is considered. There is a project management model given for which the domain experts IT defined a standardised relational positional pattern $A_{R W S}$ shown in the following matrix: 


$A_{R W S}=\left[\begin{array}{llllll}0 & 1 & 0,2 & 0,7 & 0,5 & 0,3 \\ 1 & 0 & 0,8 & 0,2 & 0,4 & 1 \\ 0,2 & 0,8 & 0 & 0,5 & 0,6 & 0,2 \\ 0.7 & 0,2 & 0,5 & 0 & 1 & 0,4 \\ 0,5 & 0,4 & 0,6 & 1 & 0 & 0,6 \\ 0,3 & 1 & 0,2 & 0,4 & 0,6 & 0\end{array}\right]$

All the obtained results are grouped into three linguistic sets defining the strength of interaction of chosen roles pairs id est lack or low $\left(\mathrm{B}_{1}\right)$, average $\left(\mathrm{B}_{2}\right)$, high $\left(\mathrm{B}_{3}\right)$. On the basis of experts' opinions a model of value division is accepted which is based on the following way of assigning to particular sets: extreme value for lack or low 0 , typical value for set average 0.5 , extreme value for set high 1 . The values which are critical points and Taken ranges will be output set models $y_{R W S}$.

The strength of interaction is determined by frequency (significance) of cooperation among roles during realisation of a project. It is assumed that frequency (significance) of cooperation will be described in three linguistic sets, id est low $\left(\mathrm{A}_{1}\right)$, average $\left(\mathrm{A}_{2}\right)$, high $\left(\mathrm{A}_{3}\right)$. On the basis of experts' opinions a points model of frequency (significance) of cooperation among roles evaluation is accepted, where 0 points is given to a pair of roles which have to collaborate in a project and 10 points to a pair of roles which have to collaborate constantly. The intervening results, from range $0-10$ are assigned to remaining intervening states. In order to design ranges the experts were asked to give opinion on which value it is difficult to assign low or average value set and analogously which one belongs to average or high set. Indicated by experts values 3 and 7 form points $x_{R W S_{k_{l}}}$ and $x_{R W S_{k_{2}}}$. The assumed ranges will form a model of output sets $x_{R W S}$.

The experts built a knowledge base defining correlation between model's states of input and output. This knowledge base include following rules:

- R1: If $x_{R W S}$ is low Then $y_{R W S}$ is lack or row

- R2: If $x_{R W S}$ is average Then $y_{R W S}$ is average

- R3: If $x_{R W S}$ is high Then $y_{R W S}$ is high.

The above mentioned model involving relational role pattern and division of its value to fuzzy sets is an image of perfect situation developed for given project management model. A project supervisor taking a candidate for position $r_{2}$ ought to define frequency of their cooperation with other project roles. Because every project, regardless of accepted methodological postulates, goes by its own principles, it is the project supervisor who delegates tasks for project roles as well as scope and method of coopera- tion between the roles. The discussed project includes following set of role pairs $P R=\left\{p r_{1}, p r_{2}, \ldots, p r_{15}\right\}$, accepting assumption (1-3), where $p r_{1}=a_{12}, p r_{2}=a_{13}, p r_{4}=$ $a_{14}, p r_{5}=a_{15}, p r_{6}=a_{16}, p r_{7}=a_{23}, p r_{8}=a_{24}, p r_{9}=a_{25}, p r_{10}=$ $a_{26}, p r_{11}=a_{34}, p r_{12}=a_{35}, p r_{13}=a_{36}, p r_{14}=a_{45}, p r_{15}=a_{46}$.

The project supervisor determined the following cooperation frequency values for the discussed project: $X_{R W S}=$ $\left\{x_{R W S, p r_{1}}=4, x_{R W S, p r_{2}}=2, x_{R W S, p r_{3}}=6, x_{R W S, p r_{4}}=2, x_{R W S, p r_{5}}=\right.$ $8, x_{R W S, p r_{6}}=3, x_{R W S, p r_{7}}=10, x_{R W S, p r_{8}}=7, x_{R W S, p r_{9}}=2, x_{R W S, p r_{10}}$ $=4, x_{R W S, p r_{11}}=5, x_{R W S, p r_{12}}=9, x_{R W S, p r_{13}}=2, x_{R W S, p r_{14}}=3$, $\left.x_{R W S, p r_{15}}=8\right\}$.

Using the above mentioned model implemented in MatLab Fuzzy Toolbox environment, it is possible to conduct the following assessment (Figure 4).

A candidate to a role $r_{2}$ is evaluated thus it is necessary to assess the strength of interactions his/her roles with other roles in a team, id est for pairs $p r_{1}, p r_{7}, p r_{8}, p r_{9}, p r_{10}$. The chosen role pairs obtained the following results: $y_{R W S p r_{1}}=0.48, y_{R W S p r_{7}}=0.796, y_{R W S p r_{8}}=0.586, y_{R W S p r_{9}}=$ $0.307, y_{R W S p r_{10}}=0.48$. Those results allow to group information about strength of interactions among separate role pairs to following sets: $p r_{1}, p r_{8}$ and $p r_{10}$ to average set, $p r_{9}$ lack or row set, and pair $p r_{7}$ to high set. It means that candidate performing a task of role $r_{2}$ will remain in the strongest interaction with employee performing role $r_{3}$. In a topological relational model exactly this pair of roles will be tested for matching in the light of MBTI profile.

\subsection{Fuzzy model for topological role pattern as- sessment}

According to MBTI method human's mental functions are divided into four groups: perceptual function, which introduces two types of perception: $\operatorname{Sensing}(\mathrm{S})$ and iNtuition $(\mathrm{N})$; judgement function which introduces two types of assessing: Thinking (T) and Feeling (F); attitude to the world function which introduces two kinds of approaches: Extroversion (E) and Introversion (I); attitude to the outer world function which introduces two kinds of attitudes: Judging $(\mathrm{J})$ and Perceiving (P).

Each basic function goes through phenomenon of subtypes proportion - every human is characterised by both subtypes of given function, but it is the predominance of one over another that classifies a man to 1 of 16 MBTI specified character groups. Extreme states such as 0:1 and 1:0 usually do not occur, which means there are no extreme extroverts or introverts. Accepting that each mental function is assessed by two subtypes, which strength val- 


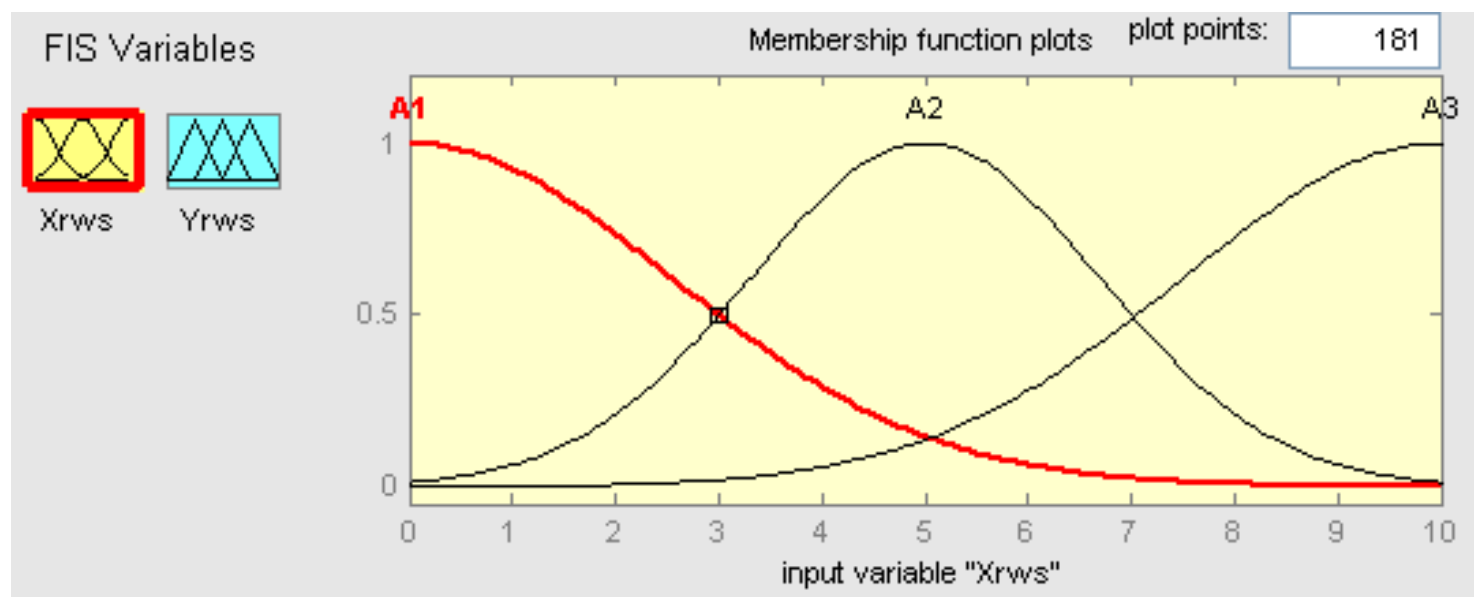

a

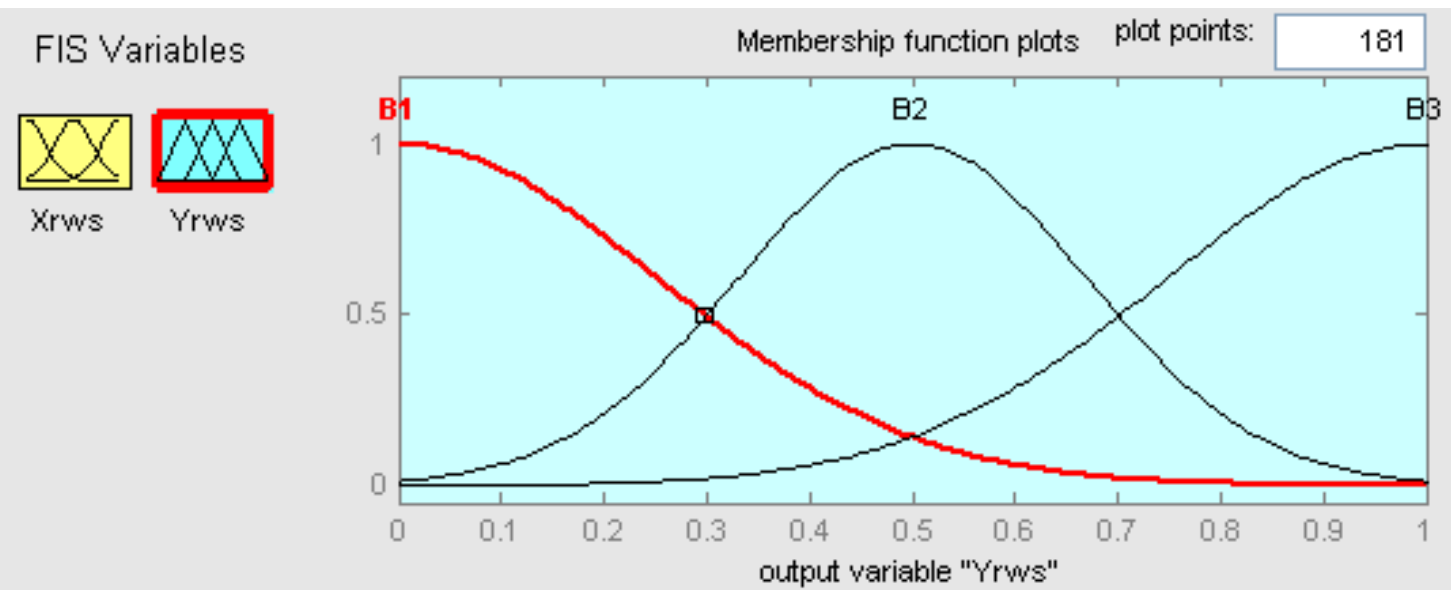

b
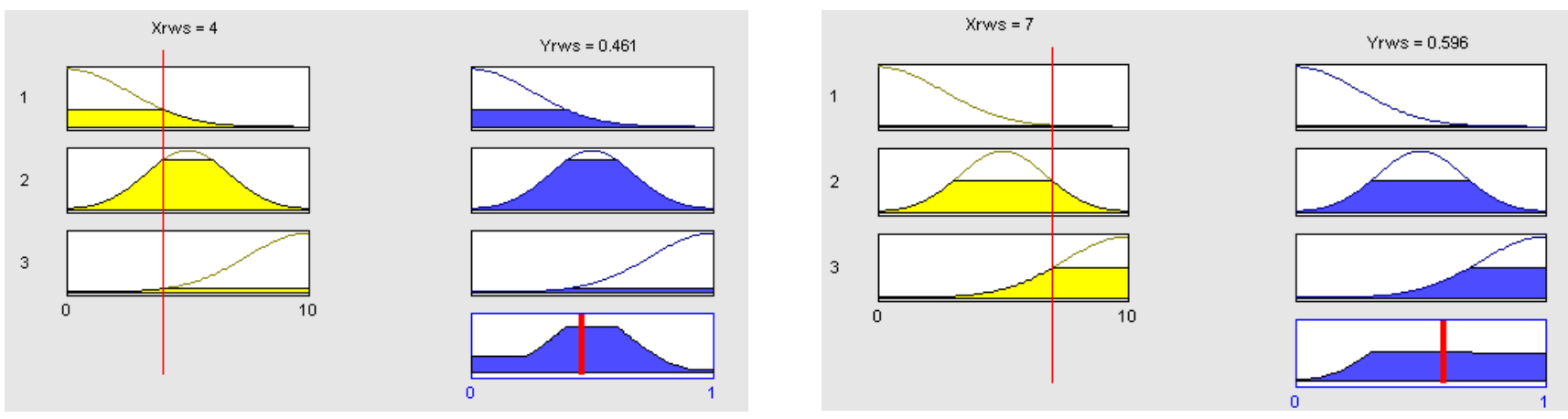

c
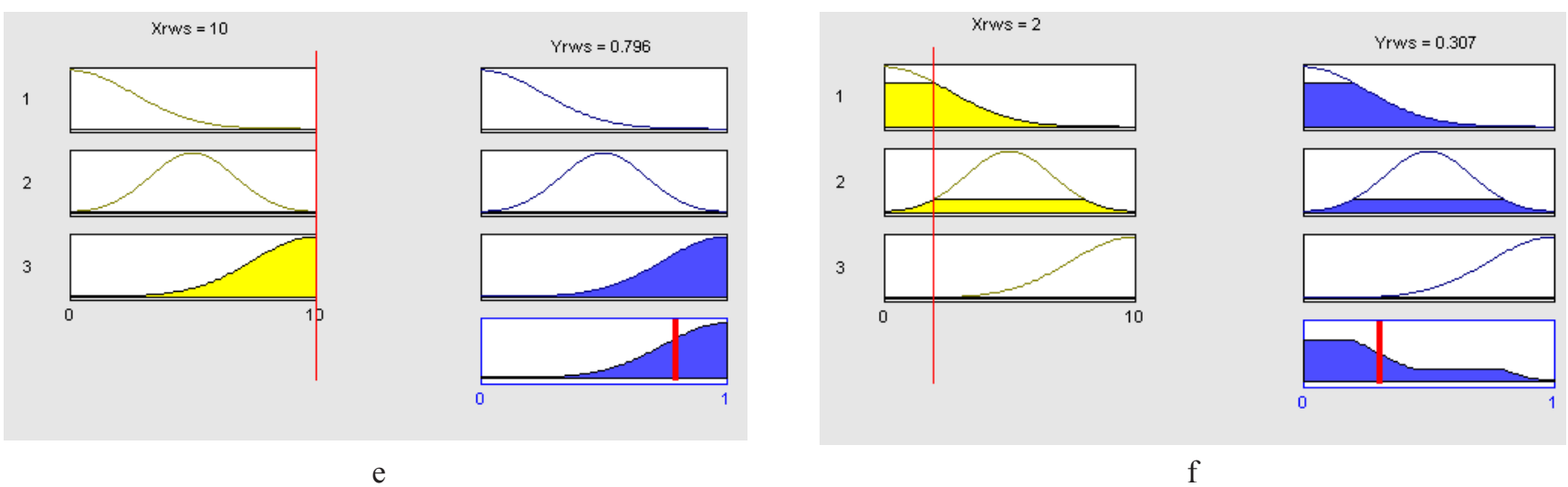

Figure 4. (a) input, (b) output, (c) results for $p r 1$ and $p r_{10}$, (d) results for $p r_{8}$, (e) results for $p r_{7}$, (f) results for $p r_{9}$. 
ue changes inversely proportionately against each other, therefore one may conclude that it is enough to evaluate the strength of only one subtype of a given function to obtain information about the second subtype of this function influence value.

For this assumption fuzzy model of topological role pattern evaluation is built in MISO (multiple input, single output) system, where model input is given linguistic variables $x_{i}$ : $\left(x_{1}-\right.$ sensual perception $\mathrm{S}, x_{2}$ - analytical assessment T, $x_{3}$ - extraversion E, $x_{4}$ - judgmental basis J), for which:

- linguistic space $X_{\mathrm{L}}$ of variables $x_{T W S}$ : \{low, average, high\};

- $\quad$ numeric space $X_{\mathrm{N}}$ of variables $x_{T W S}=\left\{x: x_{T W S} \in \mathbb{Z}, 0 \leq\right.$ $x_{T W S} \leq \max$ pt.),

- linguistic space sets are described by membership functions:

$$
\begin{array}{r}
\mu_{\text {low }}\left(x_{T W S}\right)=\frac{1}{1+e^{-a\left(x_{T W S}-b\right)},} \\
\mu_{\text {avarage }}\left(x_{T W S}\right)=e^{-\left(\frac{x_{T W S}-b}{a}\right)^{2}}, \\
\mu\left(x_{T W S k_{1}}\right)=0.5, \\
\mu\left(x_{T W S k_{2}}\right)=0.5, \\
\mu_{\text {high }}\left(x_{T W S}\right)=\frac{e^{-a\left(x_{T W S}-b\right)}}{1+e^{-a\left(x_{T W S}-b\right)},}
\end{array}
$$

and linguistic variable $r_{i}$ - project role, $r_{i}=\left\{r_{1}, \ldots, r_{n}\right\}$, for which sets defining linguistic space of variable appear as singletons, $\mu\left(r_{i}\right)=1$; at the model's output adjustment $y_{T W S}$ of desirable topological role pattern wti for chosen project role $r_{i}$ will be obtained, for which:

- linguistic space $Y_{\mathrm{L}}$ of variable $y_{T W S}:\{$ unacceptable, acceptable, suitable\};

- numeric space $Y_{\mathrm{N}}$ of variables $y_{T W S}:\left\{\mathrm{y} \in \mathbb{R}, 0 \leq y_{T W S}\right.$ $\leq 1\}$,

$$
A_{T W S}=\left[\begin{array}{lllllll}
0,2 & 0,1 & 0,5 & 0,9 & 1 & 0,3 & 0,7 \\
0,9 & 0,5 & 0,8 & 0,6 & 0,2 & 0,4 & 0,5 \\
0,6 & 0,8 & 0,6 & 0,9 & 0,1 & 0,8 & 0,2 \\
0,4 & 0,3 & 0,1 & 0,7 & 0,1 & 0,2 & 0,6 \\
0,9 & 0,7 & 0,3 & 0,2 & 1 & 0,4 & 0,9 \\
0,6 & 0,5 & 0,8 & 1 & 0,2 & 0,4 & 09
\end{array}\right.
$$

Obtained results are grouped into three linguistic sets defining a match of personal type to project role for considered roles, id est unacceptable $\left(\mathrm{F}_{1}\right)$, acceptable $\left(\mathrm{F}_{2}\right)$, suitable $\left(\mathrm{F}_{3}\right)$.On the basis of the experts' opinions a value division model was accepted. This model is based on the following values assignment to respective sets: extreme value for unacceptable 0 , typical value for acceptable 0.5 , extreme value for set suitable 1 . The values which are
- linguistic space sets of output variable are described by membership functions:

$$
\begin{gathered}
A_{T W S}=\left[\begin{array}{ccc}
a_{11} & \ldots & a_{1 n} \\
\ldots & \ldots & \ldots \\
a_{m 1} & \ldots & a_{m n}
\end{array}\right] \Rightarrow \\
\mu_{\text {unacceptable }}\left(y_{T W S}\right)=\frac{1}{1+e^{-a\left(y_{T W S}-b\right)},} \\
\mu_{\text {acceptable }}\left(y_{T W S}\right)=e^{-\left(\frac{y_{T W S}-b}{a}\right)^{2}} \\
\mu\left(y_{T W S k_{1}}\right)=0.5, \\
\mu\left(y_{T W S k_{2}}\right)=0.5, \\
\mu_{\text {suitable }}\left(y_{T W S}\right)=\frac{e^{-a\left(y_{T W S}-b\right)}}{1+e^{-a\left(y_{T W S}-b\right)}}
\end{gathered}
$$

Similarly to strength of role interactions model the process of modelling sets of system output uses standardised role relation pattern $A_{T W S}$ as well as Gauss' function with critical points $k$.

A knowledge base is represented as If - Then type of rule including all combinations of input variable values and respective set of conclusions.

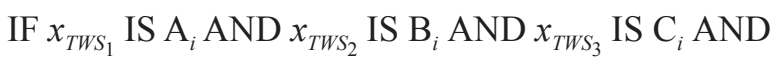

$$
\begin{aligned}
& x_{T W S_{4}} \text { IS D } \mathrm{D}_{i} \text { AND } r_{i} \text { IS E } \mathrm{E}_{i} \text { THEN } x_{T W S} \text { IS F }{ }_{i}
\end{aligned}
$$

Example 2

This example covers evaluation of candidate's match in terms of his/her personality type compatibility with prepared topological pattern built by occupational psychology experts. Following Example 1, a candidate performing a task assigned to role $r_{2}$ is evaluated. The occupational psychology experts $E p$ built matrix $A_{T W S}$ for the set of all project roles according to assumptions (5-7), including results of matching personality types $P T=\left\{p t_{1}, \ldots, p t_{16}\right\}$ to each of six project roles.

$\left.\begin{array}{ccccccccc}0,2 & 0,4 & 0,7 & 0,8 & 0,3 & 0,6 & 0,2 & 0,1 & 0,6 \\ 0,1 & 0,5 & 0,2 & 1 & 0,3 & 0,7 & 0,1 & 0,8 & 0,2 \\ 0,3 & 0,8 & 0,6 & 0,4 & 0,5 & 0,7 & 0,7 & 0,2 & 0,4 \\ 0,8 & 0,7 & 0,4 & 0,5 & 0,9 & 1 & 0,5 & 0,3 & 0,4 \\ 0,6 & 0,1 & 0,5 & 0,4 & 0,1 & 0,8 & 0,7 & 0,5 & 0,3 \\ 0,7 & 0,1 & 0,6 & 0,8 & 1 & 0,2 & 0,4 & 0,6 & 0,2\end{array}\right]$

critical points $y_{T W S_{k 1}}=0.4$ and $y_{T W S_{K_{I}}}=0.6$. Taken ranges will be output set models .

Assessing candidate's personal type requires running tests which result in numerical outcome for four psychological functions groups and in each of them for two of those functions types. Accepting the assumption from Section 6.2, fuzzy description needs assessments of only four subtypes, id est S, T, E, J (alternatively N, F, I, P). 
Above subtypes are fuzzy model input variables. Every input variable $x i$ has the same model of linguistic sets description, id est grouping values into three sets low $\left(\mathrm{A}_{1}, \mathrm{~B}_{1}, \mathrm{C}_{1}, \mathrm{D}_{1}\right)$, average $\left(\mathrm{A}_{2}, \mathrm{~B}_{2}, \mathrm{C}_{2}, \mathrm{D}_{2}\right)$ and high $\left(\mathrm{A}_{3}\right.$, $B_{3}, C_{3}, D_{3}$ ), which are defined by sigmoidal and Gauss's functions. There are critical points for output functions - respectively 0 as minimum for outer left and 50 for outer right, typical value for average range is 25 . Gauss's critical points are determined $x_{T W S_{k r T}}=20$ and $x_{T W S_{k r r}}=30$. Accepted ranges are input set model. Additionally the system's input is given variable $r=\left\{r_{1}, \ldots, r_{6}\right\}$, defining which role from the set of project roles $R$ is evaluated in the light of desirable MBTI character of personal profile. Variable $r_{i}$ is described by fuzzy sets in form of singleton $\left(\mathrm{E}_{1}, \ldots, \mathrm{E}_{6}\right)$.

There is a knowledge base built for accepted model, including following rules:

$$
\begin{gathered}
\text { IF } x_{T W S_{1}} \text { IS A } A_{i} \text { AND } x_{T W S_{2}} \text { IS B B AND } x_{T W S_{3}} \text { IS C }_{i} \text { AND } \\
x_{T W S_{4}} \text { IS D D AND } r_{i} \text { IS E E THEN } x_{T W S} \text { IS } F_{i}
\end{gathered}
$$

In a quoted example, the knowledge base has 486 rules describing correlations between system input sets and designed output.

Assuming that considered candidate's test's results are: $\mathrm{S}=24, \mathrm{~T}=36, \mathrm{E}=16, \mathrm{~J}=40$, for system's input one needs to give value $x_{1}=25, x_{2}=36, x_{3}=16, x_{4}=40$ and $r_{i}=r_{2}$.

Model's implementation in MatLab environment, using Mamdany's model, max-prod inference mechanism and sharpening based on the centre of a gravity model allows to get result $y_{T W S}=0.36$ which means candidate's MBTI profile, described as INTJ from indicated characteristics, is acceptable result for a given project role. Next step is to determine whether INTJ personal type will harmonise with personal type of an employee acting as role $r_{3}$, for which there is a strong interaction level with role $r_{2}$.

\subsection{Fuzzy model for topological-relational role pattern assessment}

Fuzzy model using topological-relational role pattern is built in SISO system, where system input is given variable $x_{i}$ : $\left(x_{1}-\right.$ personality type of recruited candidate, $x_{2}$ - personality type of a candidate whose role has strong interaction with recruited candidate) so that:

- consideration space $X$ of variables $x_{T R W S 1}$ and $x_{T R W S 2}$ : $\left\{p t_{1}, \ldots, p t_{16}\right\}$,

- each personal type is described by singleton type of set for $x_{\text {TRWS }}: \mathrm{A}_{1}, \ldots, \mathrm{A}_{16}$, for $x_{\text {TRWS }}: \mathrm{B}_{1}, \ldots, \mathrm{B}_{16}$, for which,

and variable $p r_{i}-$ pair of project roles $p r_{\mathrm{i}}=\left\{p r_{1}, \ldots, p r_{15}\right\}$, for which sets describing linguistic space of variable are formed as singletons $\left(\mathrm{C}_{1}, \ldots \mathrm{C}_{15}\right)$, for which $\mu\left(p r_{i}\right)=1$

and at model's output one will obtain value of variable $y_{\text {TRWS }}-$ matching of desirable topological patterns, for which:

- linguistic space $Y_{\mathrm{L}}$ of variable $y_{\text {TRWS }}$ : \{unacceptable, acceptable, suitable\},

- numeric space $Y_{\mathrm{N}}$ of variable $y_{T R W S}:\left\{y_{T R W S} \in \mathbb{R}, 0 \leq y_{T R W S}\right.$ $\leq 1\}$,

- linguistic spaces sets of output variable are described by membership functions:

$$
\begin{gathered}
A_{\text {TRWS }}=\left[\begin{array}{ccc}
a_{11} & \ldots & a_{1 n} \\
\ldots & \ldots & \ldots \\
a_{n 1} & \ldots & a_{n m}
\end{array}\right] \\
\mu_{\text {unacceptable }}\left(y_{T R W S}\right)=\frac{1}{1+e^{-a(y-b)},} \\
\mu_{\text {acceptable }}\left(y_{T R W S}\right)=e^{-\left(\frac{y-b}{a}\right)^{2}}, \\
\mu\left(y_{T R W S k_{1}}\right)=0.5, \\
\mu\left(y_{T R W S k_{2}}\right)=0.5, \\
\mu_{\text {suitable }}\left(y_{T R W S}\right)=\frac{e^{-a(y-b)}}{1+e^{-a(y-b)}}
\end{gathered}
$$

Analogously to above mentioned models also here the process of modelling systems output sets uses standardised position relation pattern $A_{\text {TRWS }}$ as well as Gauss' function with critical points $k$.

A knowledge base is represented as If - Then type of rule including all combinations of input variable values and respective set of conclusions.

$$
\begin{gathered}
\text { IF } x_{T R W S_{1}} \text { IS A AND } x_{T R W S_{2}} \text { IS B B AND } p r_{i} \text { IS C } C_{i} \text { THEN } \\
x_{\text {TRWS }} \text { IS } \mathrm{D}_{i}
\end{gathered}
$$

\section{Example 3:}

Continuing thoughts from Example 1, in effect of interaction strength assessment between role $r_{2}$ of considered/ recruited candidate and other roles in a team a conclusion is drawn that it is role $r_{3}$ that remains in the strongest interaction with role $r_{2}$. For this pair of roles $p r_{7}$ it is necessary to check the topological match of people who are employed to get through them.

Let us assume that a candidate recruited to role $r_{2}$ has ISTJ character type, id est $p t_{1}$, but a person employed for role $r_{3}$ has ESFP character type, id est $p t_{10}$. In order to assess the quality of those two types it is necessary to use built, standardised topological-relational matrix of role patterns $A_{T R W S}$ developed by occupational psychology experts $E p$. Matrices $A_{\text {TRWS }}$ are built for every set of roles in a given project management model $A_{T R W S_{i}}=\left\{A_{T R W S_{1}}, \ldots, A_{T R W S_{n}}\right\}$. In a quoted example for defined role pair $p r_{7}$ the experts built a relational-topological matrix, for values which 
were grouped into three linguistic sets defining topological matching of considered roles, id est unacceptable $\left(\mathrm{D}_{1}\right)$, acceptable $\left(\mathrm{D}_{2}\right)$, suitable $\left(\mathrm{D}_{3}\right)$. On the basis of experts' opinions a model of value division is accepted which is based on the following way of assigning to particular sets: extreme value for unacceptable 0 , typical value for set acceptable 0.5 , extreme value for set suitable 1 . The values which are critical points $y_{T R W S_{k 1}}=0.4$ and $y_{T W S_{k 2}}=$ 0.6 . Taken ranges will be output set models $y_{T W S}$.
The knowledge base enabling to assess topological match includes 9215 rules which stems from an amount of sets describing input variables $\left(x_{1}-16\right.$ sets, $x_{2}-16$ sets, $p r_{i}$ - 36 sets) which describe all topological combinations for all accepted pairs of roles, accepting assumptions (5) and (6).

For a chosen role pair $p r_{7}$ a result is obtained: $y_{T R W S, p r_{1}}=$ 0.69 , which means that this result classifies match of those

$$
A_{\text {TRWS }}=\left[\begin{array}{cccccccccccccccc}
0,9 & 0,8 & 0,6 & 0,6 & 0,8 & 0,8 & 0,6 & 0,6 & 1 & 0,9 & 0,7 & 0,7 & 0,9 & 0,9 & 0,6 & 0,7 \\
0,7 & 0,6 & 0,4 & 0,4 & 0,6 & 0,6 & 0,4 & 0,4 & 0,8 & 0,7 & 0,5 & 0,5 & 0,7 & 0,7 & 0,4 & 0,5 \\
0,6 & 0,5 & 0,3 & 0,3 & 0,5 & 0,5 & 0,3 & 0,3 & 0,7 & 0,6 & 0,4 & 0,4 & 0,6 & 0,6 & 0,3 & 0,4 \\
0,6 & 0,5 & 0,2 & 0,3 & 0,5 & 0,5 & 0,2 & 0,3 & 0,6 & 0,6 & 0,3 & 0,4 & 0,6 & 0,6 & 0,3 & 0,3 \\
0,8 & 0,7 & 0,5 & 0,5 & 0,7 & 0,7 & 0,5 & 0,5 & 0,9 & 0,8 & 0,6 & 0,6 & 0,8 & 0,8 & 0,5 & 0,6 \\
0,6 & 0,5 & 0,3 & 0,3 & 0,5 & 0,5 & 0,3 & 0,3 & 0,7 & 0,6 & 0,4 & 0,4 & 0,6 & 0,6 & 0,3 & 0,4 \\
0,6 & 0,5 & 0,3 & 0,3 & 0,5 & 0,5 & 0,3 & 0,3 & 0,7 & 0,6 & 0,4 & 0,4 & 0,6 & 0,6 & 0,3 & 0,4 \\
0,6 & 0,5 & 0,2 & 0,3 & 0,5 & 0,5 & 0,2 & 0,3 & 0,6 & 0,6 & 0,3 & 0,4 & 0,6 & 0,6 & 0,3 & 0,3 \\
0,9 & 0,8 & 0,6 & 0,6 & 0,8 & 0,8 & 0,6 & 0,6 & 1 & 0,9 & 0,7 & 0,7 & 0,9 & 0,9 & 0,6 & 0,7 \\
0,8 & 0,7 & 0,5 & 0,5 & 0,7 & 0,7 & 0,5 & 0,5 & 0,9 & 0,8 & 0,6 & 0,6 & 0,8 & 0,8 & 0,5 & 0,6 \\
0,6 & 0,5 & 0,2 & 0,3 & 0,5 & 0,5 & 0,2 & 0,3 & 0,6 & 0,6 & 0,3 & 0,4 & 0,6 & 0,6 & 0,3 & 0,3 \\
0,6 & 0,5 & 0,2 & 0,3 & 0,5 & 0,5 & 0,2 & 0,3 & 0,6 & 0,6 & 0,3 & 0,4 & 0,6 & 0,6 & 0,3 & 0,3 \\
0,8 & 0,7 & 0,5 & 0,5 & 0,7 & 0,7 & 0,5 & 0,5 & 0,9 & 0,8 & 0,6 & 0,6 & 0,8 & 0,8 & 0,5 & 0,6 \\
0,8 & 0,7 & 0,4 & 0,5 & 0,7 & 0,7 & 0,4 & 0,5 & 0,8 & 0,8 & 0,5 & 0,6 & 0,8 & 0,8 & 0,5 & 0,5 \\
0,6 & 0,5 & 0,2 & 0,3 & 0,5 & 0,5 & 0,2 & 0,3 & 0,6 & 0,6 & 0,3 & 0,4 & 0,6 & 0,6 & 0,3 & 0,3 \\
0,7 & 0,6 & 0,3 & 0,4 & 0,6 & 0,6 & 0,3 & 0,4 & 0,7 & 0,7 & 0,4 & 0,5 & 0,7 & 0,7 & 0,4 & 0,4
\end{array}\right]
$$

two personal types to suitable set. That means a candidate performing tasks for role $r_{2}$ and employee with role $r_{3}$ have compatible characters in terms of possibility for an effective cooperation.

\section{$7 \quad$ Summary}

This paper shows fragmentary models in chosen aspects that allow to design processes of candidate's assessment for project position. Those models are appropriate for assessment and match of single candidate to already existing project team or for designing one that consists of new candidates. The quoted method of assessment postulates verification of three main aspects: (a) evaluation of candidate's match in terms of compatibility of MBTI personal type with adopted topological pattern designed by occupational psychology experts, (b) indication of which roles a candidate will remain in strong interactions with and (c) assessment of candidate's topological match for a given role with personal type of people playing roles which remain in strong interaction with candidate's role.
All those measures aim at streamlining project team's cooperation quality through providing coherence of competence and character.

Using fuzzy logic tool for model description seems to be natural, due to necessity of considering information supplied by the experts and information concerning aspects of soft competencies described in words, based on intuition and domain experts' empiric knowledge, in decisional process.

It is vital to take into consideration the aspect of possible implementation of various kinds of fuzzy sets representations for description of decisional variables and the impact of representation choice on quality (preciseness/ adequacy) of acquired results in taken tasks.

This paper aims at expounding the sheer idea of applying role patterns in which further stages become foundation for modelling of expected outputs of fuzzy system model in process of recruitment and selection of employees for project teams. 


\section{$8 \quad$ References}

[1] Babuska R. - Fuzzy modeling - a control engineering perspective. Proc. Inter. Conf. Fuzz-IEEE/IFES'95, Yokohama, Japan 1995, pp. 1897-1902.

[2] Cakrt M. - Kto jest kim. Typy osobowiści dla menedżerów. One Press, Gliwice 2006.

[3] Chapko M. - O zwinnym zarzadzaniu projektami. Helion, Gliwice 2013.

[4] Cobb Ch.G. - Making Sense of Agile Project Management: Balancing Control and Agility. APN Promise SA, Warszawa 2012.

[5] Cockburn A. - Agile Software Development: The Cooperative Game. (Second Edition), Helion, Gliwice 2008.

[6] Forsyth D.R. - Group dynamics. (Second edition), Pacific Grove, CA: Brooks/Cole 1990.
[7] Hannay J.E. et al - Effects of personality on Pair Programming. Software Engineering, IEEE Transactions on Vol. 36, No. 1, Jan-Feb 2010, pp. 61-80.

[8] Piegat A. - Modelowanie i sterowanie rozmyte. Wydawnictwo EXIT, Warszawa 2003.

[9] Rasmusson J. - The Agile Samurai: How Agile Masters Deliver Great Software. Helion, Gliwice 2012.

[10] Schaber K. - Agile Project Management with Scrum. Microsoft Press, Warszawa 2005.

[11] Wojnar J. - Multicriteria decision making model for the new team members selection based on individual and group-related factors. [in] Foundation of Management, Vol. 3, No. 2 (2011), pp. 106-108.

[12] http://pmresearch.pl/ Raport z Badania Polskich Projektów IT 2010 